\section{Why can't people be more like bats?}

\section{Cheating Monkeys and Citizen Bees: The Nature of Cooperation in Animals and Humans \\ by Lee Dugatkin}

Free Press: 1999. 174 pp, \$25, £16.99

\section{Laurent Excoffier}

What have hermaphroditic sea bass, Mother Teresa and naked mole rats in common? This is not the latest dirty joke, but rather a serious issue treated in Lee Dugatkin's latest book Cheating Monkeys and Citizen Bees. These are three of the many organisms cited as displaying examples of cooperative behaviour, without which any society would, sooner or later, crumble. In order to avoid this fatal outcome, Dugatkin suggests, we should study the evolution of animal cooperation, because such a study "can be used to help us better understand and promote human cooperation". As animals present behaviours that are free of the influence of human culture, they can give us clues about cooperative strategies where moral will is absent, and where the only judge is natural selection.

Like four pillars of wisdom, Dugatkin describes four different strategies of cooperation: family dynamics, in which one is more likely to help relatives than strangers; reciprocal transactions, in which individuals cooperate with those they trust; selfish teamwork, in which cooperation can often be seen as a mere by-product of a selfish behaviour; and group altruism, in which individual altruistic behaviour (even though sacrificial) may be maintained when the unit of selection is the group where it belongs. This segmentation does not mean that animal cooperative behaviours are always so simple that they must necessarily belong to one of these categories, but these "four paths to cooperation" are based on sufficiently different evolutionary mechanisms to justify their distinction.

Each chapter is richly illustrated by wellchosen and often illuminating examples of cooperative behaviours, such as the baby-sitting behaviour of dwarf mongooses, the regurgitation of blood meals in vampire bats for their starving roostmates, the formation of coalition to rapt females in baboons, the exploratory tour of guppy fishes in front of predators, the helping behaviour of beeeater birds towards their baby brothers and sisters, and the better-known social behaviours of ants, termite or bees. In all these cases, the author presents a very credible evolutionary explanation for the emergence and preservation of those behaviours by natural selection. Then, in an altruistic manner, he turns to the question of using this newly acquired knowledge to foster cooperation in humans.

This is where the book goes off course. The potential applications of animal models of cooperation to humans are often appallingly simplistic, and mainly consist of a collection of recipes to improve the efficiency of teamwork. Police officers, soldiers, fire-fighters and work colleagues are the main groups he thinks could improve their cooperative behaviour. He suggests various measures to achieve this aim. Army or police units should be structured around relatedness, for example, since kin are more willing to help each other in dangerous situations. Small teams of related individuals would work harder because of kin-related benefits. Pairs of police officers should be teamed for long periods to increase their chances of interacting and helping each other. Reducing the size of teams could remove any temptation to cheat by working less.

As this book is targeted for a lay audience these ideas may well attract attention from people delighted (or afraid) to find some biological justification for nepotism or the creation of harsher working environments.

The book is a mixture of well-presented behavioural ecology and cheap sociology. Though Dugatkin is a recognized behavioural ecologist, he is far from being an outstanding philosopher. The human applications mentioned above almost all deal with people exposed to some sort of pressure (war, thieves, fire or work), where some penalty is imposed on cheats (death or a pay cut). The author's mistake is to transpose natural selection, a powerful explanatory paradigm in animals, into a mere coercive force in humans, implying that cooperation can be increased by additional constraints. Will the spirit of 1984 rule the twenty-first century? Probably, if we try to foster cooperation rather than harmony.

Laurent Excoffier is in the Department of Anthropology and Ecology, University of Geneva, CP511 1211 Geneva 24, Switzerland.

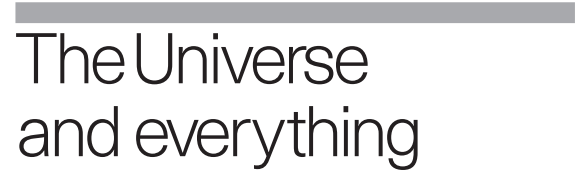

\section{Cosmological Physics}

by John A. Peacock

Cambridge University Press: 1999. 694 pp.

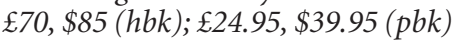

\section{Ray Carlberg}

In the 1920s, a pair of discoveries - one observational and one theoretical launched the modern era of cosmology. Hubble found his redshift distance relation, and Einstein's theory of general relativity was found to have either expanding or contracting solutions for a uniform, isotropic Universe.
By itself, either discovery would have been impressive. But the conjunction of a bold application of physical theory to the entire Universe and an observational fact was truly astonishing. There was plenty of immediate confusion, given the rather young Universe implied by Hubble's erroneous distance scale and a number of alternative physical models. More recently, a perhaps even deeper excitement has surrounded the application of new ideas in quantumfield theory and particle physics to the problems of the very early Universe and its relation to the existence of matter and structure. This new conjunction of physics and astrophysics offers tests of particle physics theory at energies far beyond any laboratory accelerator.

The intermingling of observational detail and fundamental theory has made cosmology an exceptionally rich, exciting and controversial science. Students in the field - whether observers or particle theorists - are expected to be acquainted with matters ranging from the Supernova Ia distance scale, Big Bang nucleosynthesis theory, scale-free quantum fluctuations during inflation, the galaxy two-point correlation function, particle theory candidates for the dark matter, and the star formation history of the Universe. Several general science books, conference proceedings and specialized monographs have addressed these issues. Peacock's Cosmological Physics ambitiously fills the void for introducing students with a strong undergraduate background in physics to the entire world of current physical cosmology. The majestic sweep of his discussion of this vast terrain is awesome, and is bound to capture the imagination of most students.

Cosmological Physics will appeal to the adventuresome, and may trouble those who like to see every detail of an argument spelled out. The avalanche of ideas rapidly, but not breathlessly, coaxes the reader straight into the excitement of the field. Peacock takes the view that an introduction to the physics of cosmology should not shield the reader from ideas and data whose significance is not yet clear. For instance, he includes brief sections on modified Newton dynamics and the anthropic principle. In spite of this, some of the observational details have already seen substantial changes in their interpretation, notably the redshift dependence of the star formation rate.

The result is an impressive overview of cosmology as a physical science. This abundance will communicate the widespread excitement of the subject as fundamental physics, and will inspire others to learn the details.

Ray Carlberg is in the Department of Astronomy,

University of Toronto, 60 Saint George Street,

Toronto, Ontario M5S 3H8, Canada. 